\title{
Correlation between Epiretinal Membrane Bridging, Visual Acuity and Central Macular Thickness
}

\author{
Jelena Paovic ${ }^{1 *}$, Predrag Paovic ${ }^{1}$ and Anka Stanojevic Paovic ${ }^{2}$ \\ ${ }^{1}$ University Eye Clinic; Clinical Center of Serbia; 11000 Belgrade; Serbia \\ ${ }^{2}$ Uvea Center, Center for Diagnosis and Treatment of Ocular Diseases; Kneza od Semberije 14; 11000 Belgrade. Serbia
}

\begin{abstract}
Epiretinal membrane or macular pucker is avascular, fibrocellular membrane that develops on the inner surface of the retina that can result in various stages of macular dysfunction. Fine epiretinal membranes lead to minimal visual loss, whilst epiretinal membranes (that may or may not bridge the fovea) are, in their more severe stages, accompanied by macular edema and image distortion that is caused by pronounced tractional membranes and perifoveal capillary leakage. Macular edema and its' type, as well as epiretinal membrane bridging the fovea can precisely be defined and monitored via optical coherence tomography.

This study included a total of 138 patients (188 eyes) with epiretinal membranes (95 on the right and 93 on the left eye). Macular edema was present in 144 eyes with, and 69 eyes without, epiretinal membranes. Data obtained for both eyes indicates that upon analysis number of patients in both examined groups (epiretinal membrane bridged and did not bridge the fovea) increases exponentially with increased visual acuity, and suggests that there is no considerable difference in distribution of visual acuity with regards to patients in both examined groups. This data also indicates that patients with the worst central macular thickness $(>500 \mu)$ are represented only in the group of patients where epiretinal membrane had bridged the fovea, but that in both examined groups there is a difference in prevalence of patients with central macular thickness of 400 to $499 \mu$ (more common in the group that did, than in the group that did not, bridge the fovea). Hence, there exists a relationship between epiretinal membrane that bridges the fovea and central macular thickness.
\end{abstract}

Aim of this study is to determine the extent in which epiretinal membrane bridging or not bridging the fovea influences visual acuity and to determine the relationship between them; central macular thickness; and visual acuity.

Keywords: Central macular thickness; Epiretinal membrane; Fibrocellular membrane; Optical coherence tomography

\section{Introduction}

Epiretinal membrane (ERM) or macular pucker is avascular, fibrocellular membrane that develops on the inner surface of the retina that can result in various stages of macular dysfunction [1]. Membrane formation is a result of complex pathophysiological mechanisms that are based on tissue damage and consequential reparation of the same [2].

Proliferation and transdifferentiation of hyalocytes that are, after posterior vitreous detachment (PVD), found in the remains of cortical gel on the surface of the retina lead to formation of an ERM [3-9]. ERM that occurs in early PVD is usually localized on the surface of the retina, below the separated posterior vitreal cortex. In large clinical studies partial or complete PVD was detected in 80 to $95 \%$ of the eyes with idiopathic ERM $[10,11]$. Though they are usually asymptomatic, early stages of PVD may be complicated by several macular pathologies and partially determined by size of residual vitreomacular adhesion [12].

Latest data obtained via electron microscopy suggests that vitreal debri, formed by separation of the vitreal cortex (vitreoschisis), can trigger pronounced fibrocellular proliferation $[6,9,13]$ which results in formation of a membrane that is made up of collagen; retinal pigment epithelium (RPE) cells; glial cells; astrocytes; fibrocytes; and macrophages $[9,14]$, and which can be found on the posterior vitreal cortex and retinal interface, and that can, but does not have to, during the evolution of ERM, bridge the fovea.

In its' nature, ERM can be idiopathic or secondary. Secondary membranes are associated with various diseases including: retinal vascular diseases (central retinal vein occlusion (RVO) or retinal vein branch occlusion); uveitis; trauma or changes (inflammation) that occur following retinal detachment surgery $[12,15]$.

According to its' severity Idiopathic ERM can be divided into several stages i.e. ERM 0; ERM 1; and ERM 2. Fine ERM lead to minimal visual loss, whilst ERM (that may or may not bridge the fovea) are, in their more severe stages, accompanied by macular edema (ME) and image distortion that is caused by pronounced tractional membranes and perifoveal capillary leakage. ME can be cystic or diffuse and membrane attachments may be diffuse or focal. Nearly $50 \%$ of patients with ERM have more than one place of retinal contraction that is accompanied by higher incidence of intraretinal cysts and significantly thicker retina.

Traction that is directed from the center to the periphery may, upon vitreoschisis, lead to macular ruptures (pseudoholes) [16]. Macular pseudohole which is accompanied by ERM is considered to be central defect in the ERM that is, during the initial stages of PVD, localized on sites of vitrofoveal adhesion.

ME and type of edema, as well as ERM bridging the fovea can

*Corresponding author: Paovic J, University Eye Clinic; Clinical Center of Serbia; 11000 Belgrade, Serbia, Tel: +381112438054/+38162261113; E-mail: ordinacija@uveacentar.com

Received July 17, 2017; Accepted August 13, 2017; Published August 20, 2017

Citation: Paovic J, Paovic P, Paovic AS (2017) Correlation between Epiretina Membrane Bridging, Visual Acuity and Central Macular Thickness. J Cytol Histol 8: 471. doi: 10.4172/2157-7099.1000471

Copyright: @ 2017 Paovic J, et al. This is an open-access article distributed under the terms of the Creative Commons Attribution License, which permits unrestricted use, distribution, and reproduction in any medium, provided the original author and source are credited. 
Citation: Paovic J, Paovic P, Paovic AS (2017) Correlation between Epiretinal Membrane Bridging, Visual Acuity and Central Macular Thickness. J Cytol Histol 8: 471. doi: 10.4172/2157-7099.1000471

precisely be defined and monitored via optical coherence tomography (OCT) [17-20]. Prospective studies indicate that in all patients with idiopathic ERM one can, with an aid of an OCT, detect partial or complete PVD [12,21,22]. Three-dimensional (3D) assesment of ERM shows that with an aid of spectral domain OCT (SD-OCT) partial or complete PVD can be detected in in up to $97 \%$ of cases [23]. Macular traction may lead to macular detachment that can sometimes be just barely visible and very shallow.

\section{Aim}

To determine the extent in which ERM, bridging or not bridging the fovea, influences visual acuity.

To determine the relationship between central macular thickness; epiretinal membranes (bridging or not bridging the fovea); and visual acuity.

\section{Materials and Methods}

This study included total of 138 patients where ERM was present on 188 eyes. Visual acuity (VA) tests are performed with full correction thus providing the so called best corrected visual acuity (BCVA) which is taken on the Snellen chart (scale values ranging between 0.1 and 1.0, ten levels) and whose results are then converted into a logarithmic scale. VA which are expressed in $\log M A R$ have then been grouped into four intervals $(\geq 1 ; 0.52-1.0 ; 0.15-0.52 ; 0-0.15)$.

Fundus examination was performed with an aid of an indirect biomicroscopic lens Super Vitreo Fundus (Volk) and a three mirror contact lens (Haag-Streit 903).

High-definition spectral domain OCT (SOCT, Copernicus+, Optopol Technology, Zawiercie, Poland) which was used in order to scan the macula had a wavelength of $830 \mathrm{~nm}$ and axial resolution of 6 $\mu$; transverse resolution between 12 and $18 \mu$; and $10 \mathrm{~mm}$ long $\mathrm{B}$ - scan with $25000 \mathrm{~A}$ - scans per second.

Patients who had ERM were, based on their appearance and volumetric changes in the retina, according to Gass classification classified into three groups (ERM 0; ERM 1; and ERM 2). From the obtained OCT data the following were assessed: ERM attachments to the retina (diffuse or focal); presence and type of edema (cystic or diffuse); and whether or not ERM bridges the fovea. Excluding criteria were patients who had no ERM; as well as their age and gender. Basic statistical processing methods, given the sample size, included frequency of occurrence of tested parameters; crossing frequency tables; and graphical representations of obtained results (i.e. in form of bar charts and precentages). Statistical analysis was performed with an aid of SPSS version 17.

Authors have obtained all necessary ethical approvals and patients' informed consent has been granted. All authors hereby declare that all data has been examined and approved by the appropriate ethics committee and performed in accordance with the ethical standards laid down in the 2008 Declaration of Helsinki. Authors have also declared that no competing interests exist nor have they received any financial compensation for it.

\section{Results}

95 patients had ERM on the right eye and 93 patients had it on the left eye. While in 70 patients $(73.7 \%)$ ERM had bridged the right eye fovea and in 25 patients (26.3\%) it did not, in 68 patients (73.1\%) ERM had bridged the left eye fovea and in 25 patients (26.9\%) it did not.
Out of the 138 patients that had ERM that had bridged the fofea the autors had randomly chosen four eyes from as many patients.

On OCT one can see a highly reflective inner surface of the retina that bridges the fovea and corresponds to an ERM. In these regions, ERM is somewhat separated from the retina. The central thickness amounts to $625 \mu$ and mean thickness to $427 \mu$ (Figure 1).

On an OCT image of the left eye one can clearly see hyperreflective layer over an uneven, split-up inner surface of the retina that bridges the fovea and corresponds to thickened, developed ERM. It is only occasionally linked to the retina and, in those places, has the greatest traction. ERM is $25 \mu$ thick and at a distance of $42 \mu$ from the retina (Figure 2).

On an OCT image of the left eye one can see that, as a result of tractional ERM, foveal contour is flattened. It can clearly be seen as a $22 \mu$ thick, highly reflective layer that bridges the fovea and which is centrally adherent to the inner surface of the retina. Retina is thickened and stratified and there is a presence of intraretinal, optically empty, spaces that correspond to cysts (Figure 3 ).

On an OCT image of the right eye one can see that there is an elevated contour of the fovea with changed retinal configuration i.e. it is centrally thicker and stratified, with accumulated fluid in the outer layers and diffuse macular edema. Due to traction of focally attached ERM (that can be seen as a highly reflective band that bridges the fovea), its inner surface appears to be a bit jagged. It is located at a distance of $18 \mu$ and is $26 \mu$ thick (Figure 4).

Of the total number of patients (50) that had ERM that does not bridge fovea, we have randomly chosen seven patients (8 eyes), of

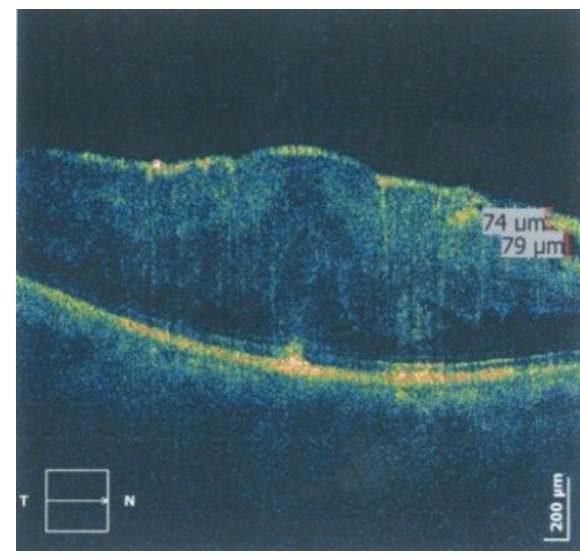

Figure 1: Optical coherence tomography image of right eye with a complete loss of foveal contour.

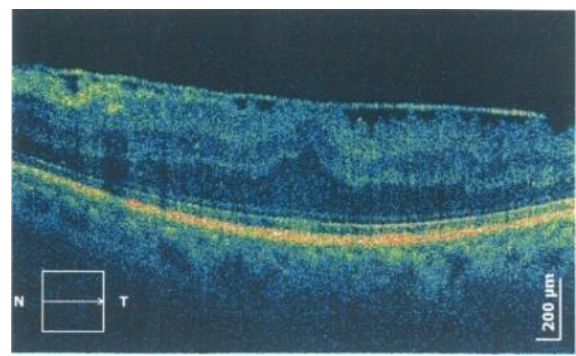

Figure 2: Optical coherence tomography image of the left eye with thickened, developed, somewhat joined, epiretinal membrane. 
Citation: Paovic J, Paovic P, Paovic AS (2017) Correlation between Epiretinal Membrane Bridging, Visual Acuity and Central Macular Thickness. J Cytol Histol 8: 471. doi: 10.4172/2157-7099.1000471

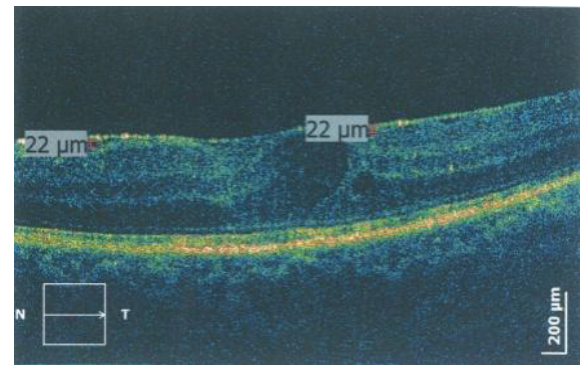

Figure 3: Optical coherence tomography image of the left eye that depicts a tractional epiretinal membrane and cysts.

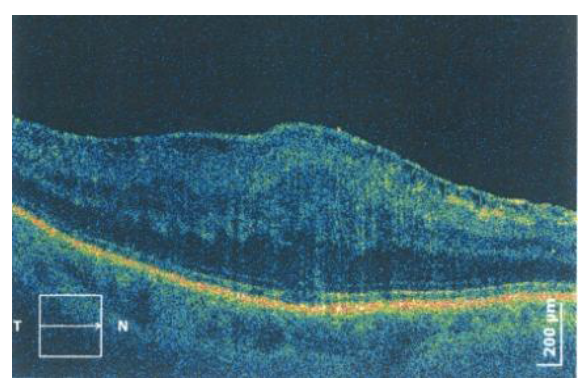

Figure 4: Optical coherence tomography image of the right eye with the presence of an epiretinal membrane which bridges the fovea and a diffuse macular edema.

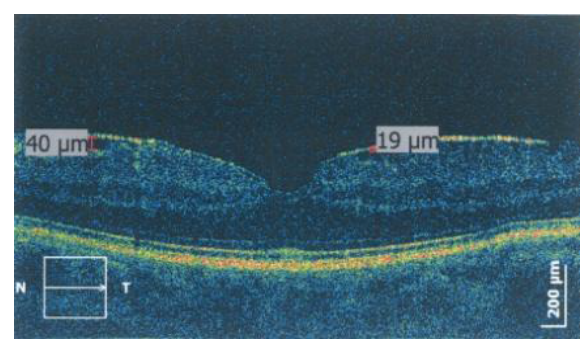

Figure 5: Optical coherence tomography image of the left eye with epiretinal membrane which doesn't bridge the fovea.

which three patients ( 3 eyes) had ERM that did not bridge the fovea, but (seeing that macular ruptures can form during the evolution of ERM) who had pseudohole and lamellar macular holes.

Left eye OCT image depicts changed foveal contour that is, due to tractional ERM which does not bridge the fovea, is steeper temporally and that thus seen as a highly reflective band along the inner surface of the retina. This membrane is $19 \mu$ thick temporally and $40 \mu$ nasally away from the centre of the retina (Figure 5).

A preserved foveal contour can be seen on the OCT image of the left eye. Nasally away from the fovea one can note a highly reflective band that corresponds to a developed, $38 \mu$ thick, discontinuous, adherent ERM which does not bridge the fovea (Figure 6).

A preserved foveal contour can be seen on the OCT image of the left eye. On the inner surface of the retina there is a reflection of an ERM that does not bridge the fovea. Central retinal thickness was 265 $\mu$ and average thickness was $239 \mu$ (Figure 7).

While an OCT image of the right eye shows a preserved foveal contour, the inner surface of the retina depicts a highly reflective thin line that corresponds to an $18 \mu$ thick ERM which does not bridge the fovea (Figure 8).

OCT image of the right eye shows nasally steeper but preserved foveal contour. Due to focal tractional ERM, that can on an OCT be seen as a thin highly reflective band, the inner surface of the retina is elevated. ERM spreads nasally away from the centre with a break which is located in the proximity of the foveola (Figure 9).

OCT image of the left eye with changed foveal contour and laterally spaced central layer that thus forms a lamellar macular hole. On the inner surface of the retina there is a highly reflective band which corresponds to a $28 \mu$ thick ERM (Figure 10).

OCT image of the left eye fovea with changed contour and a characteristic appearance of a pseudohole that has a temporally steeper edge. Inner surface of the retina depicts a highly reflective band that corresponds to a $27 \mu$ thick, foveally discontinuous ERM (Figure 11).

OCT of the right eye with changed, elevated, foveal contour and

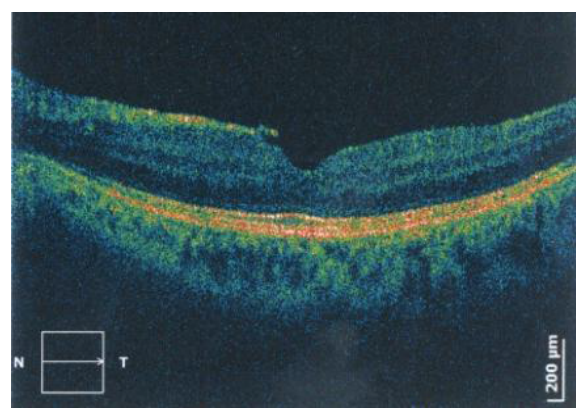

Figure 6: Optical coherence tomography image of the left eye with discontinuous epiretinal membrane that doesn't bridge the fovea.

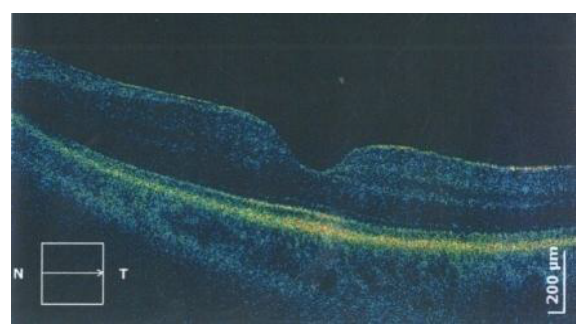

Figure 7: Optical coherence tomography image of the left eye with an epiretinal membrane that doesn't bridge the fovea.

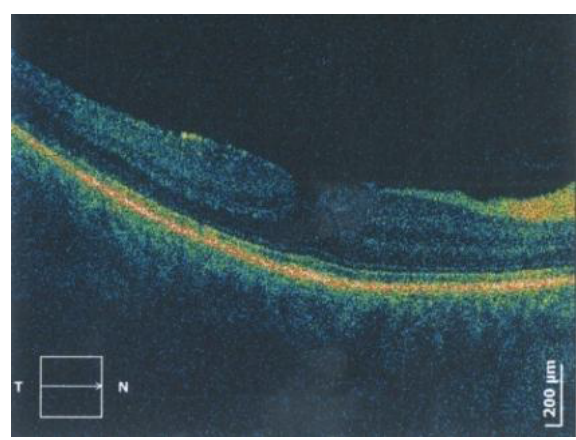

Figure 8: Optical coherence tomography image of the right eye with an epiretinal membrane that doesn't bridge the fovea. 
Citation: Paovic J, Paovic P, Paovic AS (2017) Correlation between Epiretinal Membrane Bridging, Visual Acuity and Central Macular Thickness. J Cytol Histol 8: 471. doi: 10.4172/2157-7099.1000471

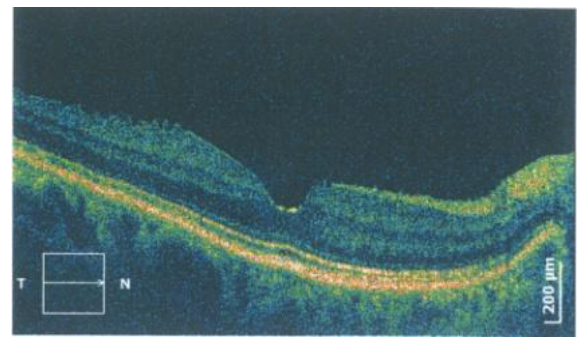

Figure 9: Optical coherence tomography image of the right eye with an epiretinal membrane that doesn't bridge the fovea.

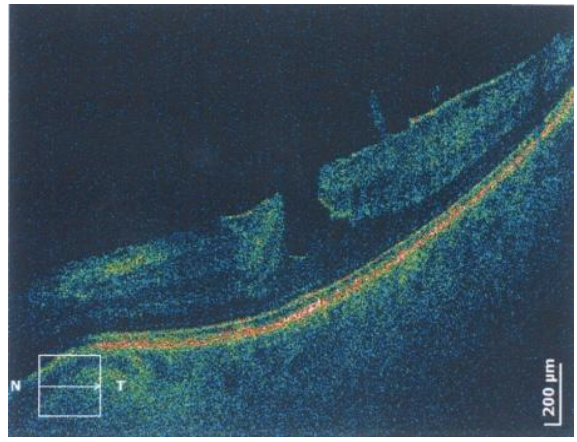

Figure 10: Optical coherence tomography image of the left eye with an epiretinal membrane that doesn't bridge the fovea and a lamellar macular hole.

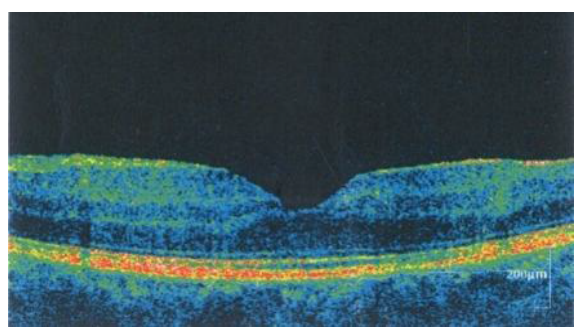

Figure 11: Optical coherence tomography image of the left eye with an epiretina membrane which doesn't bridge the fovea and a pseudohole.

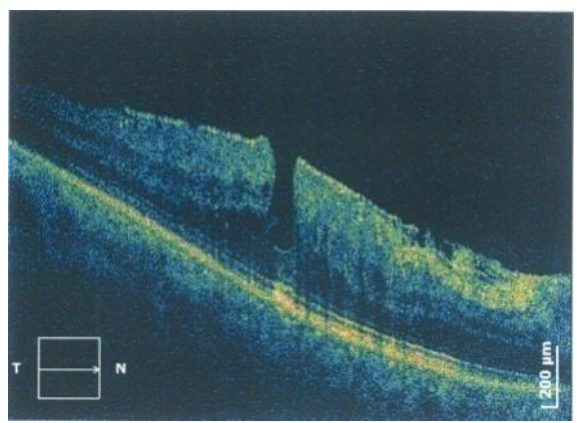

Figure 12: Optical coherence tomography image of the right eye with an epiretinal membrane which doesn't bridge the fovea and a pseudohole.

characteristic pseudohole. On the inner surface of the retina one can clearly observe a highly reflective band that corresponds to a $20 \mu$ thick ERM. Nasally from the fovea, this membrane is partially separated from the retina $(46 \mu)$ and thus the inner surface is, in places of attachment, somewhat elevated (Figure 12).

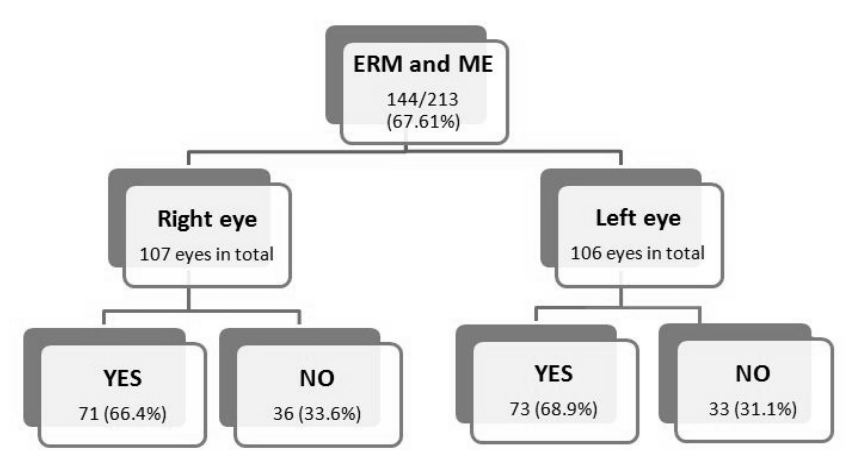

Description ERM - Epiretinal membrane; ME - macular edema.

Figure 13: Macular edema and epiretinal membrane.

Investigation into the presence of an ME related to patients who had ERM; presence and absence of ME can be seen in Figure 13.

Comparison between data which was obtained for both eyes indicates that the number of patients in both examined groups (ERM bridges and does not bridge the fovea) increases exponentially with an increase in VA. This data suggests that there is no considerable difference in distribution of VA with regards to patients with ERM that bridges or does not bridge the fovea (Figure 14a and 14b).

Comparison of the data obtained for the right and left eye indicates that patients with the worst central macular thickness (CMT) $(>500$ $\mu$ ) are represented only in the group of patients where ERM bridges the fovea. Results also indicate that in both groups (with and without bridging the fovea) there is a difference in prevalence of patients with CMT 400-499 $\mu$ (more common in the group that bridges fovea). These results indicate that relationship exists between ERM, which bridges the fovea and the CMT (Figure 15a and 15b).

\section{Discussion}

Macular ERM are avascular proliferations of benign glial cells that have epicentric contractible macular or paramacular expansion usually located somewhat under the posterior pole. Contraction of a membrane results in wrinkling of the macula which leads to functional impairment. Although their pathophysiology is not completely known, they are chronologically associated with an occurrence of PVD and their incidence increases with age [24].

On an OCT, ERM can be seen as a highly reflective band or a line on the inner surface of the retina. Foveal contour changes are, to a greater or lesser extent, due to tractional ERM. They can be highly adherent to the retina and thus difficult to visualize. Once they are partially separated from the retina, it is possible to clearly define zones of adherence and the inner surface of the retina which appears to be indented and uneven.

Clinical appearance of ERM depends on its' thickness and size of contraction which leads to wrinkling (folding). Thin membranes are usually asymptomatic and transparent and result in limited contraction and thus formation of radial, striated, folds of the internal limiting membrane (ILM) and the inner layers of the retina ('distortion of the inner surface of the retina') starting from the edge of the ERM or the epicentre of the contraction. Thicker, highly contracted membrane places tractional stress on all the layers of the retina that, with the development of an edema, leads to extensive macular dysfunction. Persistent macular traction can lead to thickening and stratification 
Citation: Paovic J, Paovic P, Paovic AS (2017) Correlation between Epiretinal Membrane Bridging, Visual Acuity and Central Macular Thickness. J Cytol Histol 8: 471. doi: 10.4172/2157-7099.1000471

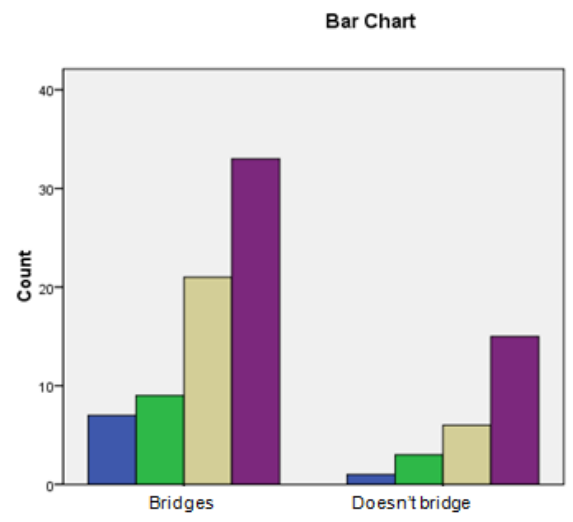

2a.ERM bridges the fovea-Right eye

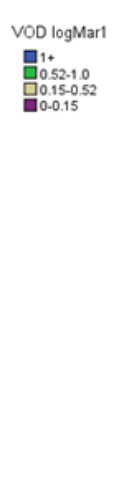

ERM - epiretinal membrane; VOD - visual acuity right eye; VOS - visual acuity left eye

Figure 14: ( $a$ and b) Comparison of visual acuity (left and right eyes) and epiretinal membranes that bridge the fovea.

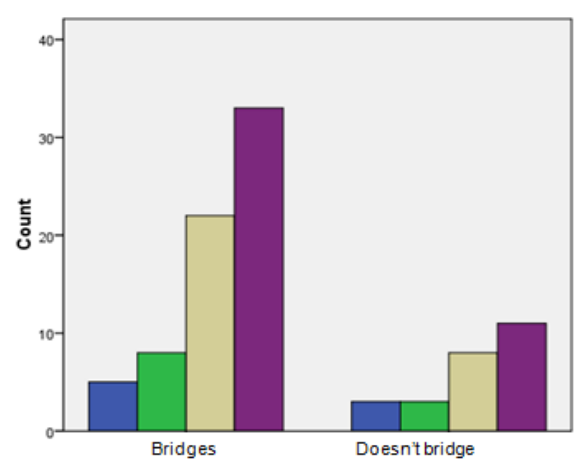

2b. ERM bridges the fovea - Left eye

\section{Bar Chart}

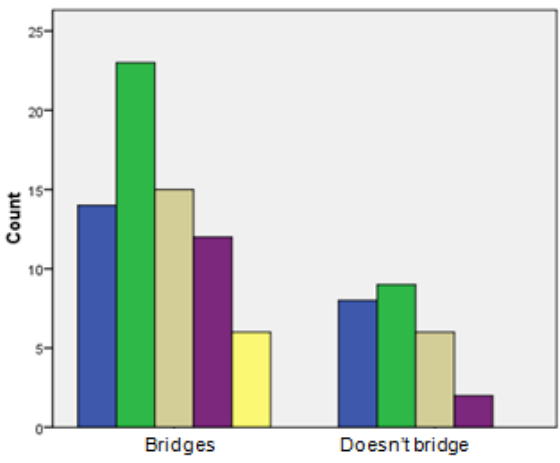

3a. ERM bridges the fovea-Right eye
Bar Chart

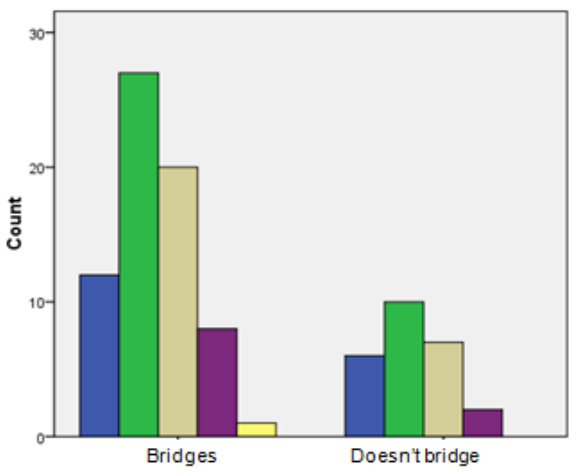

3b. ERM bridges the fovea- Left eye

ERM - epiretinal membrane; CMT - central macular thickness

Figure 15: $(a$ and $b)$ Comparison between central macular thickness findings and epiretinal membranes that bridge the fovea.

of the retina with intraretinal fluid accumulation (cystoid or diffuse edema) and withdrawal of central foveal layers.

ERM does not have to bridge the fovea, but in case that it does, it can be associated with ME. Results of this study indicate that ERM which bridges the fovea was 2.5 times more prevalent than discontinuous ERM that does not bridge the fovea. These results also show that there is a noteworthy relationship between ERM which bridges the fovea and bigger CMT.

Patients with good VA had the lowest CMT. Even though data obtained from literature suggests that VA is associated with CMT, seeing that larger CMT was associated with patients who had ERM that bridged the fovea and that was at the same time independent of VA, it is deemed that, instead of CMT, it is actually the preservation of the photoreceptor layer which is of the utmost importance for the VA [25].

\section{Conclusion}

Limiting factors of studies such as this can be associated with resolution of an OCT (higher being the better one) that is used for identification and monitoring of various changes. On the other hand, when comparing VA and ERM one should take into account that both traction and other factors may influence ME, and thus visual functions (ME and ERM in case of uveitis; diabetes mellitus; and etc.).

Although comparisons that were performed on results obtained for ERM that bridged and didn't bridge the fovea and VA suggest that there exists no relationship between them but rather that VA depends on CMT, one should take into account that presence of ERM sustains tractional ME. Obtained results are important for clinical practice in that they contribute and guide the practitioner in decision making concerning future treatment, wich may or may not, include ERM pilling (surgical removal of an ERM) as well as decrease of tractional ME and thus a decrease of CMT.

\section{References}

1. Gandorfer A, Haritoglou C, Scheler R, Schumann R, Zhao F, et al. (2012) Residual cellular proliferation on the internal limiting membrane in macular pucker surgery. Retina 32: 477-485.

2. Jaffe NS (1967) Macular retinopathy after separation of vitreoretinal adherence. Arch Othalmol 78: 585-591.

3. Gass JDM (1997) Stereoscopic atlas of macular diseases: diagnosis and treatment (4thedn), St. Louis: Mosby 4: 904-951.

4. Kishi S, Demaria C, Shimizu K (1986) Vitreous cortex remnants at the fovea after spontaneous vitreous detachment. Int Ophthalmol 9: 253-260. 
Citation: Paovic J, Paovic P, Paovic AS (2017) Correlation between Epiretinal Membrane Bridging, Visual Acuity and Central Macular Thickness. J Cytol Histol 8: 471. doi: 10.4172/2157-7099.1000471

5. Doi N, Uemura A, Nakao K, Sakamoto T (2005) Vitreomacular adhesion and the defect in posterior vitreous cortex visualized by triamcinolone-assisted vitrectomy. Retina 25: 742-745.

6. Gandorfer A, Rohleder M, Grosselfinger S, Haritoglou C, Ulbig M, et al (2005) Epiretinal pathology of diffuse diabetic macular edema associated with vitreomacular traction. Am J Ophthalmol 139: 638-652.

7. Kita T, Hata Y, Kano K, Miura M (2007) Transforming growth factor-2 and connective tissue growth factor in proliferative vitreoretinal diseases. Possible involvement of hyalocytes and therapeutic potential of rho kinase inhibitor. Diabetes 56: 231-238.

8. Yamashita T, Uemura A, Sakamoto T (2008) Intraoperative characteristics of the posterior vitreous cortex in patients with epiretinal membrane. Graefes Arch Clin Exp Ophthalmol 246: 333-337.

9. Schumann RG, Schaumberger MM, Rohleder M, Haritoglou C, Kampik A, et al. (2006) Ultrastructure of the vitreomacular interface in full-thickness idiopathic macular holes: a consecutive analysis of 100 cases. Am J Ophthalmol 141 1112-1119.

10. Dupas B, Tadaioni R, Erginay A, Massin P, Gaudrich A (2009) Subfoveal deposits secondary to idiopathic epiretinal membranes. Opthalmology 116: 1794-1798.

11. Dupas B. Tadajoni R (2012) Membranes epiretiniennes maculaires. EMCOphthalmology 9: 1-1.

12. Johnson MW (2005) "Perifoveal vitreous detachment and its macular complications". Trans Am Ophthalmol Soc 103: 537-567.

13. Chang LK, Fine HF, Spaide RF, Koizumi H, Grossniklaus HE (2008) Ultrastructural correlation of spectral-domain optical coherence tomographic findings in vitreomacular traction syndrome. Am J Ophthalmol 146: 121-127.

14. Bottós J, Elizalde J, Arevalo JF, Rodrigues EB, Maia M (2012) Vitreomacular traction syndrome. J. Ophthalmic Vis. Res 7: 148-161.

15. Kishi S, Shimizu K (1994) Oval defect in detached posterior hyaloid membrane in idiopathic preretinal macular fibrosis. American Journal of Ophthalmology 118: $451-456$.

16. Sebag J, Gupta P, Rosen RR (2007) Macular holes and macular pucker: the role of vitreoschisis as imaged by optical coherence tomography/scanning laser ophthalmoscopy. Trans Am Ophthalmol Soc 105: 121-129.

17. Kinoshita T, Kovacs KD, Wagley S, Arroyo JG (2011) Morphologic differences in epiretinal membranes onocular cohhernce tomography as a predictive factor for surgical outcome. Retina 31: 1692-8.

18. Gaudric A, Aloulou Y, Tadajoni R, Massin P (2013) Macular pseudohole with lamellar cleavage of their age remain pseudoholes. Am J Othalmol 155: 733-742.

19. Suh MH, Seo JM, Park KH, Yuhg (2009) Association between macular findings by optical coherence tomography and visual outcomes after epiretinal membrane removal. Am J Opthalmol 147: 473-480.

20. Inoue M, Morita S, Watanable Y, Kaneko T, Yamane S, et al. (2010) Inner segment/outer segment junction assessed by spectyral domen-optical coherence tomography in patients with idiopathic epiretinal membrane. Am J Opthalmol 150: 834-839.

21. Bu SC, Kuijer R, Li XR, Hooymans JM, Los LI (2014) Idiopathic epiretinal membrane. Retina 34: 2317-2335.

22. Chung SE, Lee J-H, Kang SW, Kim YT, Lee SW (2011) Characteristics of epiretinal membranes according to the presence or absence of posterior vitreous detachment. Eye (Lond) 25: 1341-1346.

23. Koizumi H, Spaide RF, Fisher YL, Freund KB, Klancnik JM Jr, et al. (2008) Three-dimensional evaluation of vitreomacular traction and epiretinal membrane using spectraldomain optical coherence tomography. Am J Ophthalmol 145: 509-517.

24. Dupas B, Tadayoni R Gaudric A (2015) Les membranes epiretiniennes maculaires. Journal Francais D' Opthalmologie 38: 861-875.

25. Ota A, Tanaka Y, Toyoda F (2016) Relationship between variations in posterior vitreous detachment and visual prognosis in idiopathic epiretinal membranes. Clinical Ophthalmology (Auckland, NZ) 10: 7-11. 